\title{
Network effects in Schelling's model of segregation: new evidences from agent-based simulation
}

\author{
Arnaud Banos \\ Géographie-Cités, CNRS/University Paris 1 and Paris/Ile-de-France Complex Systems \\ Institute (ISC-PIF), France \\ arnaud.banos@parisgeo.cnrs.fr
}

\begin{abstract}
According to two recent studies, Thomas Schelling' model of segregation is only weakly affected by the underlying spatial structure, whatever its complexity. Such a conclusion is important from an urban planning perspective as it suggests that only a very restricted range of possible actions, if any, would be able to contribute limiting social segregation, unless individual preferences are significantly modified.

Our own simulations show that, using appropriate graph-based spatial structures, one can reveal significant spatial effects and thus provide alternative planning insights. Cliques in networks indeed play a significant role, reinforcing segregation effects in Schelling's model. Introducing a small amount of noise in the model permits us to reveal more precisely this effect, without modifying the global behavior of the initial model. Furthermore, we show how a logistic model describes in a concise but precise way this global behavior at an aggregated level.
\end{abstract}

\section{Keywords}

Agent based modeling; Cliques; Networks; Schelling; Segregation

\section{Introduction}

Thomas Schelling' model of social segregation (Schelling, 1969; 1978) is certainly one of the most debated models in the social sciences ${ }^{1}$. Imagine two colored groups of individuals, living on a chessboard city, and having the capacity to change their place of living according to the color of their neighbors. Schelling demonstrated that if individuals have a mild preference for living near people of their own color, and if they move to satisfy their preference ${ }^{2}$, complete segregation at the city scale may occur.

Its capacity to demonstrate, in a simple and elegant way, how interdependent but noncoordinated local residential decisions may lead to unexpected social segregation surely explains the large success of this model. While scientists from various disciplines, ranging from social sciences (Clark and Fossett, 2008; Fossett and Dietrich, 2009; Laurie and Jaggi, 2003) and economics (Fagiolo et al, 2007; Pancs and Vriend, 2007) to physics (Dall'Asta et al, 2008; Gauvin et al, 2009; Stauffer and Solomon, 2007; Vinkovic and Kirman, 2006) and

\footnotetext{
${ }^{1}$ For example, an advanced search of JASSS text using « Schelling » as a keyword points out 83 references on JASSS website.

${ }^{2}$ Schelling's model is very limited in its scope at it is only based on individual preferences and thus does not take into account two other mechanisms at stake in social segregation, as identified for long by sociologists: discriminative processes (occurring at the institutional and/or individual level) and socio-economic factors.
} 
mathematics (Pollicot and Weiss, 2001; Zhang, 2004) handled this model, trying to find links with their own concepts and models, their conclusions all converged to Schelling initial finding: segregation may occur at the city level even if every single agent is tolerant enough (mild preference) to accept an integrated pattern.

According to two recent studies (Fagiolo et al, 2007; Fossett and Dietrich, 2009), Schelling's model of segregation is so robust that it is only weakly affected by the underlying urban structure: whatever the size and shape of city, this undesired emerging phenomenon will happen. According to other authors (Clark and Fossett, 2008), individual preferences are evolving so slowly that no real improvement can be expected in a near future. Such conclusions may have important public policy and urban planning implications as they suggest that only a very restricted range of possible actions, if any, would be able to contribute limiting social segregation. However, the situation seems even worse than expected. Indeed, we can draw two complementary conclusions from our own simulations: actual and planned geometries of cities not only accelerate and reinforce Schelling' segregation processes but they may also favor intolerant behaviors. Therefore, fatalism should be banned: public policy and urban planning have their role to play in the more than ever urgent quest for sustainable cities.

\section{Graph-based cities}

The shape of an urban area and the way it is fed by the road network are two major ways that differences occur at the intra-urban level. There are also generally very marked differences in accessibility between the centre and the different suburbs. Moreover, certain areas or neighborhoods can be told apart by their relative impermeability: gated communities and ghettos can thus be defined as cliques, which in the network theory jargon describe subsets made up of nodes adjacent to one another. What can be demonstrated is that network shape in general and cliques in particular may play a significant role in the dynamic of Schelling' model, cliques acting as local attractors, or segregation "traps".

Let us illustrate this point with four urban networks increasingly hierarchised, defined as: a) regular (grid), b) random, c) scale-free ${ }^{3}$, and d) fractal (Sierpinski tree). These last two networks may appear a bit "exotic" to the non-specialist but in fact they share more similarities with real cities than regular or random networks. For example, Bin Jiang (2007) demonstrated on a large sample of 40 US cities of different sizes that urban street networks based on street-street intersection display a scale-free property. Furthermore, fractal patterns are often presented as relevant for urban systems, when it comes to imagining more livable and sustainable cities (Batty, 2008; Frankhauser, 2008).

These four networks could have been compared directly, as in (Fagiolo et al, 2007), but we believe this would have introduced a severe bias linked to the varying degree distribution. Indeed, as evaluation of preferences by each agent is based on the proportion of his $n$ occupied neighbors having a given color, the de facto weight of each neighbor is $1 / n$ and therefore small neighborhoods are more sensitive to changes than larger ones. To avoid this problem, we arbitrarily fix the number of neighbors: each node is connected to its $n$ closest nodes, as defined by a Floyd routing algorithm (Floyd, 1962). The resulting neighboring graphs obtained then present an interesting effect: they are more or less marked by the existence of cliques.

${ }^{3}$ Generated following preferential attachment rules as proposed in (Barabasi and Albert, 1999). See (Song et al, 2006) for evidence on the non fractal topology of such networks. 


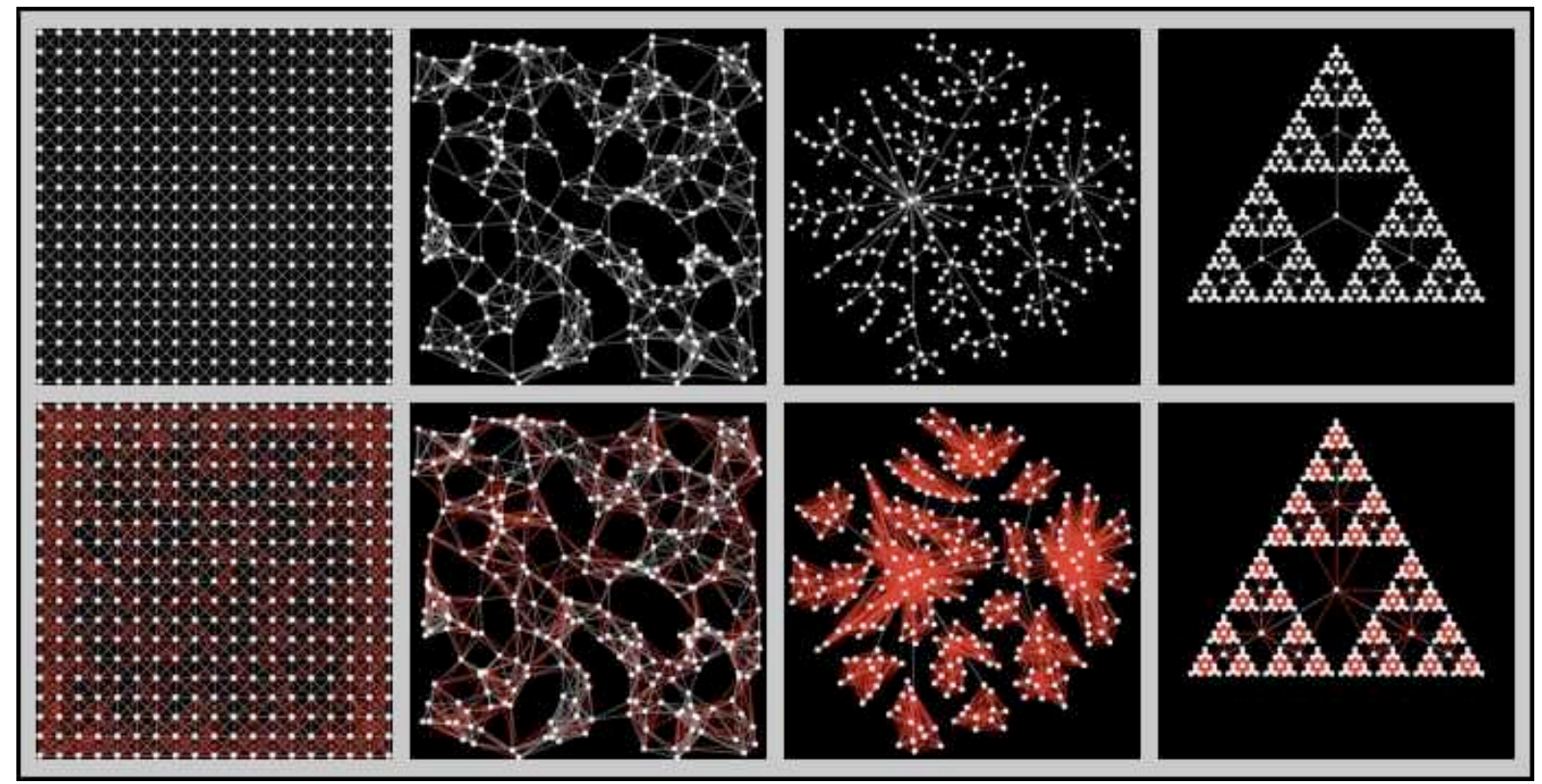

Figure 1. Regular, random, scale-free and fractal (Sierpinski) networks (up) and their corresponding neighboring graphs (bottom) for a fixed degree $(d=10$; neighborhood defined using shortest path algorithm)

As the number of neighbors $n$ is constant, each resulting network can be characterized by a simple clustering coefficient ${ }^{4}$ (Watts and Strogatz, 1998):

$$
C_{i}=\frac{2 E_{i}}{k_{i}\left(k_{i}-1\right)}, 0 \leq C_{i} \leq 1
$$

where $E_{i}$ is the number of connected pairs among neighbors of node $i$ and $k_{i}$ is the degree of node $i$.

This indicator varies between 0 (no connected pairs among neighbors of node $i$ ) and 1 (fully connected neighbors). Averaging these local values provides a global indicator very useful when comparing different network structures:

$$
\bar{C}=\frac{1}{n} \sum_{i=1}^{n} C_{i}
$$

The four networks defined, characterized by a fixed degree (10 neighbors), present different clustering values, as expected from their neighboring graphs (table 1).

\footnotetext{
${ }^{4}$ See (Newman et al, 2001) for a discussion on the limitation of this indicator when the degree varies and their proposal for a more robust indicator.
} 


\begin{tabular}{|l|c|c|c|c|}
\cline { 2 - 5 } \multicolumn{1}{c|}{} & Grid & Random & Scale-Free & Sierpinski \\
\hline \#Nodes & 361 & 361 & 361 & 363 \\
\#Edges (structural graph) & 1332 & 1448 & 360 & 364 \\
\#Edges (neighboring graph) & 2039 & 1749 & 1960 & 2134 \\
Clustering (neighboring graph) & 0.55 & 0.65 & 0.99 & 0.95 \\
\hline
\end{tabular}

Table 1. Properties of the four networks and their neighboring graphs $($ degree $=10)$

\section{Schelling's agents}

According to Schelling's model, each node of these graphs may be seen as a possible living place, occupied (or not) by an agent belonging to a given category. It is well known, however, that density of agents needs to be nor too low nor too high for the system to get self-organised (Gauvin et al, 2009; Vinkovic and Kirman, 2006). Too many vacant nodes would limit the contacts between agents, keeping their spatial distribution random whatever their individual preference level, while too few free nodes would just "freeze the system". For each generated network, a fixed proportion $(80 \%)$ of nodes is then randomly populated with a corresponding number $m$ of agents, half of them being of color $x$ and the remaining part being of color $y$. Each agent is able to identify his neighborhood composition, i.e. the proportion $P_{i j}$ of his occupied neighbors being unlike him. At each time step, each randomly selected agent $A_{i}$ computes his utility, defined as a step function:

$$
U_{i}=\left\{\begin{array}{l}
0 \text { if } \mathrm{P}_{\mathrm{ij}}>\lambda \\
1 \text { if } \mathrm{P}_{\mathrm{ij}} \leq \lambda
\end{array}\right.
$$

with $\lambda$ a tolerance threshold value, embedding agent's preference. Agent $A_{i}$ then moves if he feels unsatisfied with his current location $\left(\mathrm{U}_{\mathrm{i}}=0\right)$ and if there is at least one vacant node randomly chosen if more than one - allowing him increasing his utility $\left(\mathrm{U}_{\mathrm{i}}=0\right.$ à $\left.\mathrm{U}_{\mathrm{i}}=1\right)$.

For various possible values of the tolerance parameter ${ }^{5} \lambda=\{0,10,20,30,40,50,60,70,80$, $90,100\}, 1000$ simulations were achieved and a social mixity index calculated. Following Schelling himself, this index is simply the mean proportion of contacts between unlike neighbors (omitting empty nodes):

$$
M=\frac{1}{|A|} \sum_{i=1}^{|A|} P_{i j}
$$

where $|A|$ is the number of agents (cardinal). This index then varies between 0 (complete segregation) and approximately 50\% (mixity). It may be noticed that a $50 \%$ mixity can be obtained either from a random distribution of agents (most probable and stable issue) or from a more integrated "chessboard" like pattern (highly improbable and unstable issue).

A simulation ends up if one of the two conditions is respected: either because the system converges towards equilibrium or because the simulation time exceeds a given threshold value (table 2). Equilibrium is obtained when each agent is satisfied with his current location, that is:

\footnotetext{
${ }^{5} \lambda$ is a proportion, expressed in percents, as it refers to a threshold value of $P_{i j}$, defined for each agent $A_{i}$ as the proportion of occupied neighbors being unlike him.
} 


$$
\sum_{i=1}^{m} U_{i}=|A|
$$

It may be noticed that static patterns (i.e. no agent moves anymore) can be obtained while no equilibrium is reached:

$$
\sum_{i=1}^{m} U_{i}<|A|
$$

(Gauvin et al, 2009) call such state a "frozen state".

Results (Figure 2) confirm the robustness of Schelling's model but also stress significant discrepancies between the various networks, thus suggesting the possible role of network hierarchy and cliques in Schelling' segregation process.

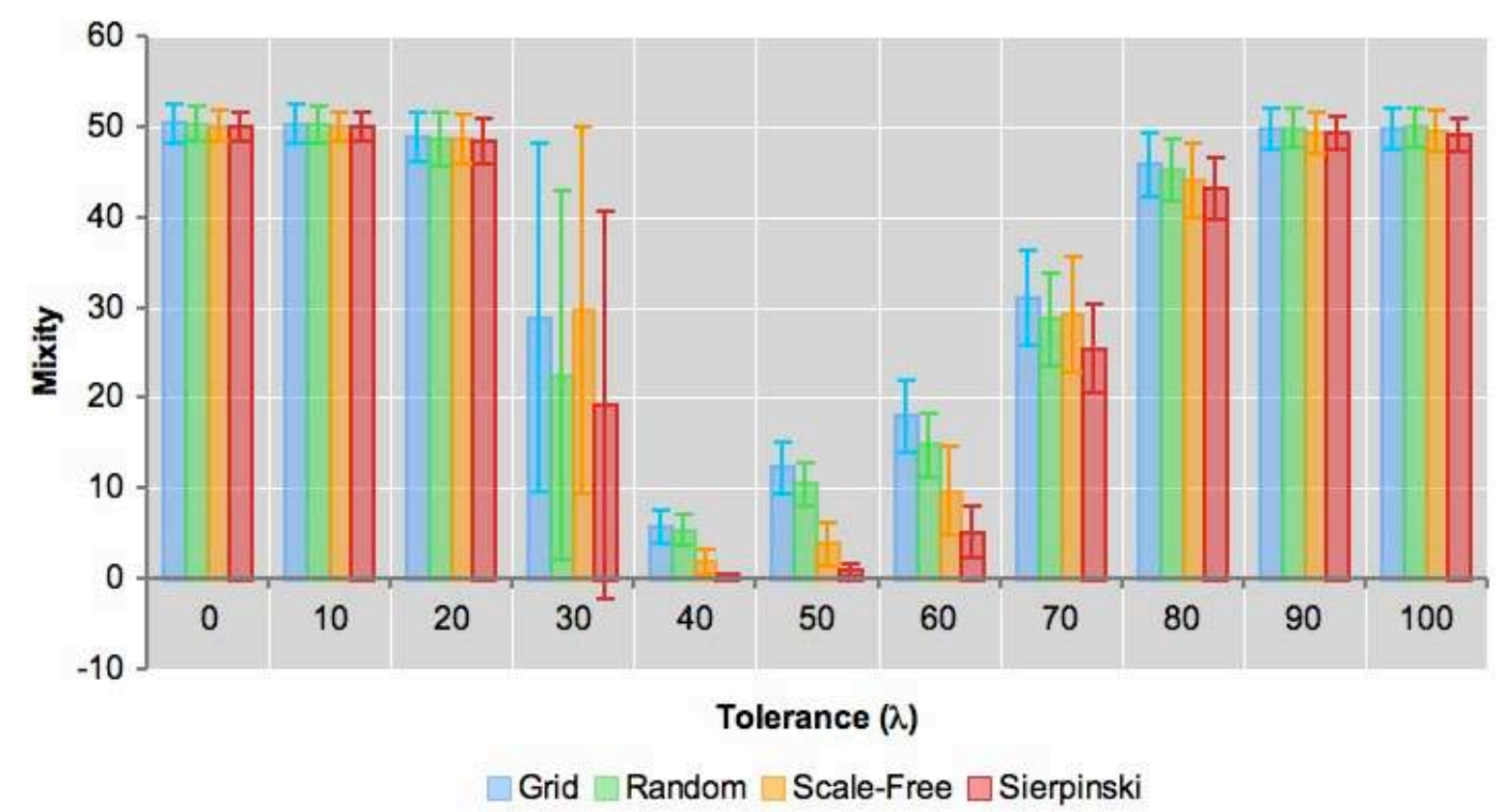

Figure 2. Average mixity index and its standard-deviation (1000 simulations), for each of the four networks

\section{Robustness of Schelling's model and cliques effect}

The typical three phases (Dall'Asta et al, 2008; Gauvin et al., 2009) of Schelling's model are clearly identified, whatever the network structure, demonstrating the robustness of this model:

- convergence towards mixed states for high tolerance values $(\lambda>70)$;

- convergence towards increasing segregated states for a large range of tolerance values $(30<\lambda<70)$

- absence of convergence for lower tolerance values $(\lambda<30)$

For high tolerance values $(\lambda>70)$, the system rapidly converges (i.e. reaches equilibrium) towards a mixed state. Then, a dramatic change occurs for a slight decrease in tolerance $(\lambda=70)$ : while being still tolerant at an individual level (preferences), agents make by their moves the global system converge towards a segregated state. As tolerance decreases $(30<\lambda<70)$, highly segregated states are obtained. For a mild preference value $(\lambda=50)$, every simulation converges from an initial random distribution to a highly segregated pattern, for each of the four generated networks (figure 3). 

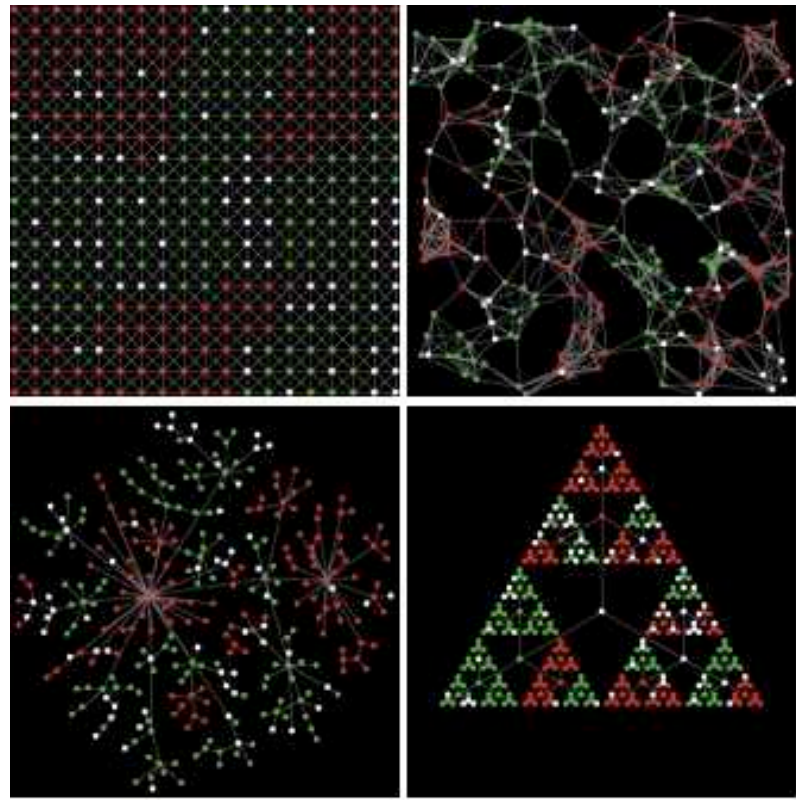

Figure 3. Examples of equilibriums attained for mild preferences $(\lambda=50$, Density $=80 \%$, Degree $=10$, white squares $=$ vacant nodes)

While having a mild preference, each agent ends up in a highly segregated community, with few or no contacts with unlike agents. Patterns are clearly related to the neighboring graphs (figure 1), underlying the importance of cliques as "segregation traps". Observing the model's dynamics confirms this role played by cliques, but also the locking-in role of "entry" nodes (figure 4). Rapidly, cliques are filled up by an agent population of one or another color. As their connection with the rest of the network is limited to a reduced number of nodes (we call them "entry" nodes), we can assume surface tension to be concentrated over this limited subset of nodes, thus reinforcing local anchorage of growing clusters. Network topology in general and cliques in particular seems to play an important role in the dynamic of the whole model, by channeling agents towards local attractors. Once these pockets of local order become filled in enough, agents are then "protected" from exogenous perturbations: no unlike agent will settle down anymore. This process may be compared to the one described by (Keeling, 1999) for epidemics, who showed how cliques reduce the initial spread of an epidemic, but also the final proportion of the population that the epidemic reaches.

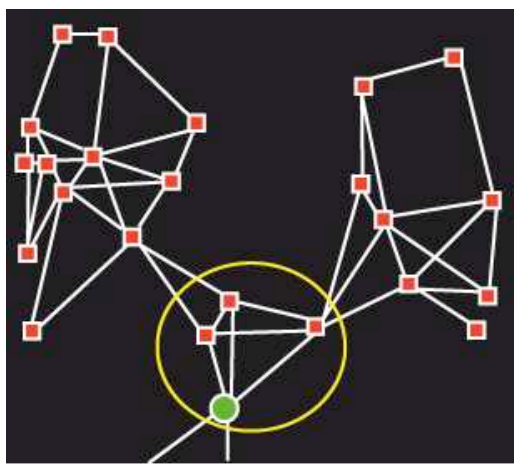

Figure 4. Cliques as segregation traps and the locking-in role played by "entry" nodes (Degree $=10$; neighboring links not represented here)

Decreasing tolerance $(\lambda=30)$ then leads to a second phase transition: agents find less and less vacant nodes corresponding to their preference and therefore wait for a better place to get vacant, while being unsatisfied with their current location. This transition area $(\lambda=30)$ is 
highly unstable and, depending on the initial configuration and the course of the simulation, "frozen states" can be reached. In such situation, the model does not converge towards equilibrium: while no agent can move anymore, a varying proportion of them are not satisfied with their current location. Table 2 shows the proportion of convergences towards equilibrium over 1000 runs, for this specific tolerance value $(\lambda=30)$. Proportions vary from $30 \%$ of the runs for grid and scale-free network, to $48 \%$ for the random network and $56 \%$ for the Sierpinski tree.

\begin{tabular}{|l|c|c|c|c|}
\cline { 2 - 5 } \multicolumn{1}{c|}{} & Grid & Random & Scale-Free & Sierpinski \\
\hline $\begin{array}{l}\% \text { convergences for } \lambda=30 \\
(1000 \text { runs })\end{array}$ & 31 & 47.8 & 32 & 55.6 \\
\hline
\end{tabular}

Table 2. Proportion of convergence towards equilibrium (each agent is satisfied with his current location) over 1000 simulations, for the $30 \%$ tolerance value and 50 iterations threshold $^{6}$

For very low tolerance values $(\lambda<30)$, no equilibrium can be found, as agents are trapped in a global frozen state, waiting forever for better places (i.e. increasing their utility) to become available.

While each of the four networks follows this global trend, significant differences occur at the two transition areas $(\lambda=70$ and $\lambda=30)$, and within the segregated state $(30<\lambda<70)$. The two hierarchised and highly clustered networks (scale-free and Sierpinski) apparently contribute reinforcing the segregation process, letting the system converge towards significantly ${ }^{7}$ higher segregation states.

Increasing the size of networks only marginally affects this very robust result. Figure 5 displays the curves for three Sierpinski networks of increasing size (respectively 364, 781 and 1464 nodes). As can be seen, the curves are very similar, whatever the network' size. However, increasing the degree (number of neighbors) significantly impacts the result.

The segregation process is indeed retarded for high tolerance values $(\lambda=70)$ while frozen states are systematically obtained for $\lambda=30$. As stated in the "Graph-based city" section, the main explanation resides in the evaluation of preferences by each agent. Indeed, as it is based on the proportion of his $n$ occupied neighbors having a given color, the weight of each neighbor is $1 / n$ and therefore small neighborhoods are much more sensitive to changes than larger ones. Increasing the degree reduces the cascading effects leading to segregation for high tolerance values $(\lambda=70)$ while it decreases to possibility for agents to find a free node corresponding to their preference for low tolerance values $(\lambda=30)$.

Such frozen states, easily obtained whenever one introduces more constraints in the system, suggest that adding some "flexibility" in the system may be interesting.

\footnotetext{
${ }^{6}$ This threshold depends both on initial conditions (network size and shape, density of agents) and simulation parameters (tolerance). However, with the decision rules defined so far, the number of iterations needed to reach convergence (being defined either as an equilibrium or as a frozen state) is usually small. In the present case, preliminary convergence studies revealed that 50 iterations is a relevant limit: if the system does not converge in 50 iterations, then its probability to find such a state in more iterations is negligible. Introducing some noise in the system, as we do in the next section, increases this value by one order of magnitude.

${ }^{7}$ Using Student't-test for comparison of sample means.
} 


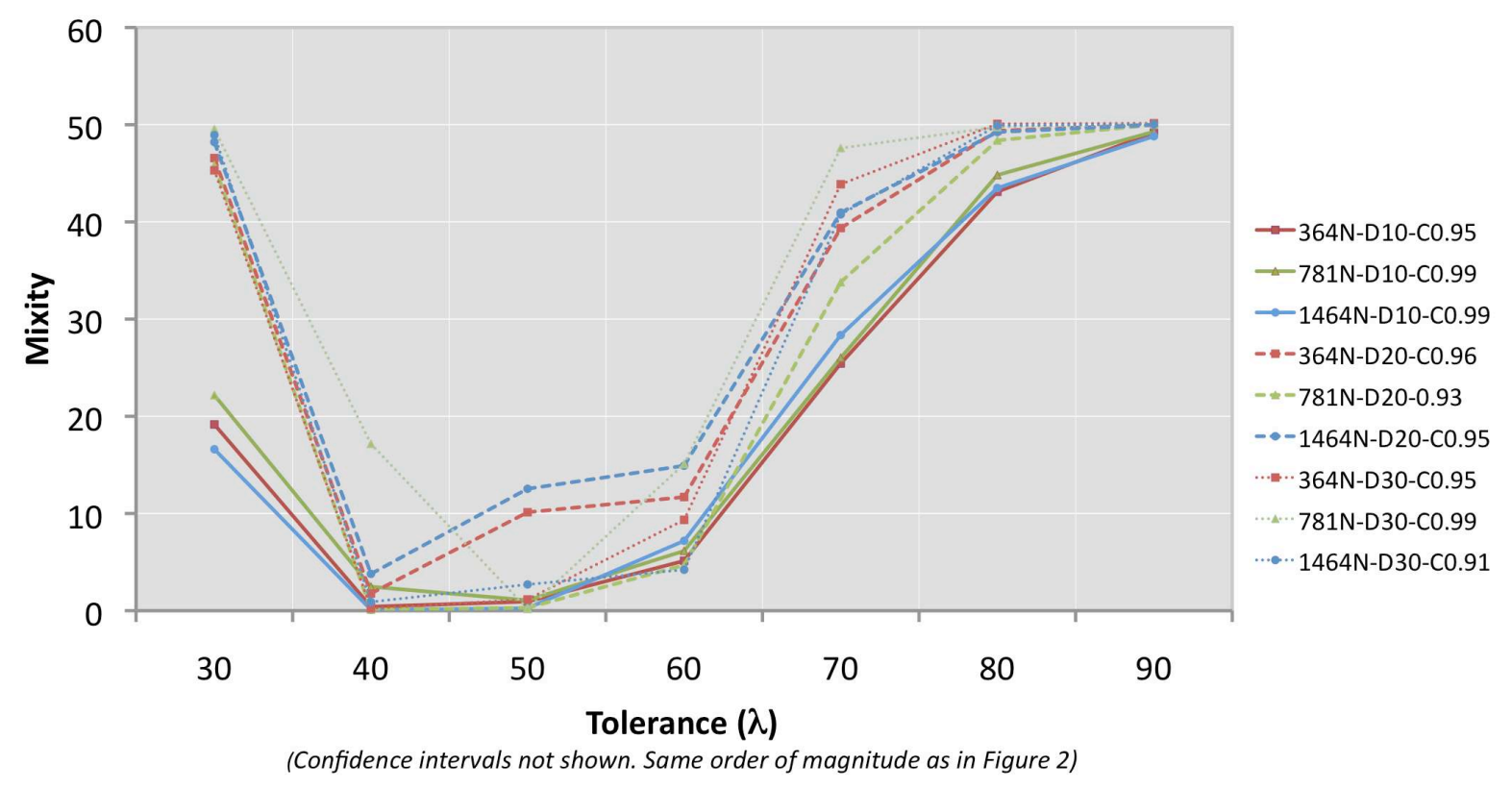

Figure 5. Comparison of results for 3 Sierpinski networks of increasing size (Average mixity index from 1000 simulations. " $N$ " is for Nodes, " $D$ " is for degree and " $C$ " is for clustering coefficient. Example: "364N-D10-C0.95" is a network with 364 nodes, a degree of 10 neighbors and a clustering coefficient of 0.95 . Confidence intervals are not shown)

\section{Varying "temperature" in Schelling's model}

One could think of a physical analogy: by "heating up" this system, we would be able to increase its entropy and therefore increase the range of possibilities for agents, as well as the range of possible states the system could reach. Adding some noise in the agents' decision rule allows reaching such objective. Several options are therefore available. For example, one can introduce the possibility for satisfied agents to also move, and not just restrict such possibility to discontent ones (Gauvin et al, 2009). As quoted by authors, "that rule introduces some noise in the dynamics and is useful to avoid a particularity of the original Schelling model, namely that the system may end up in states where the clusters are large but finite, so that strictly speaking no large-scale segregation occurs". Another option is to introduce the possibility of "mistakes" (Zhang, 2004): agents willing to move may choose a destination decreasing their utility, for example because they have incomplete or twisted information on the new context they face. It is this last option we follow here, by introducing a new parameter $N$ (for "Noise"), ranging from 0 to 1 and interfering with the decision process in the following way.

For each unsatisfied agent $A_{i, U i=0}$, we generate a random number $n$ from a uniform distribution $[0 ; 1]$ and use the following rule: if $n<N$, then agent $A_{i}$ will move to a randomly chosen vacant node, whatever its expected utility value on that destination node $\left(U_{i}=0 \rightarrow U_{i}=1 O R\right.$ $\left.U_{i}=0\right)$. Introducing even a small amount of noise $(\mathrm{N}=0.1)$ considerably "fluidifies" the system, in a very striking way (figure 6). 


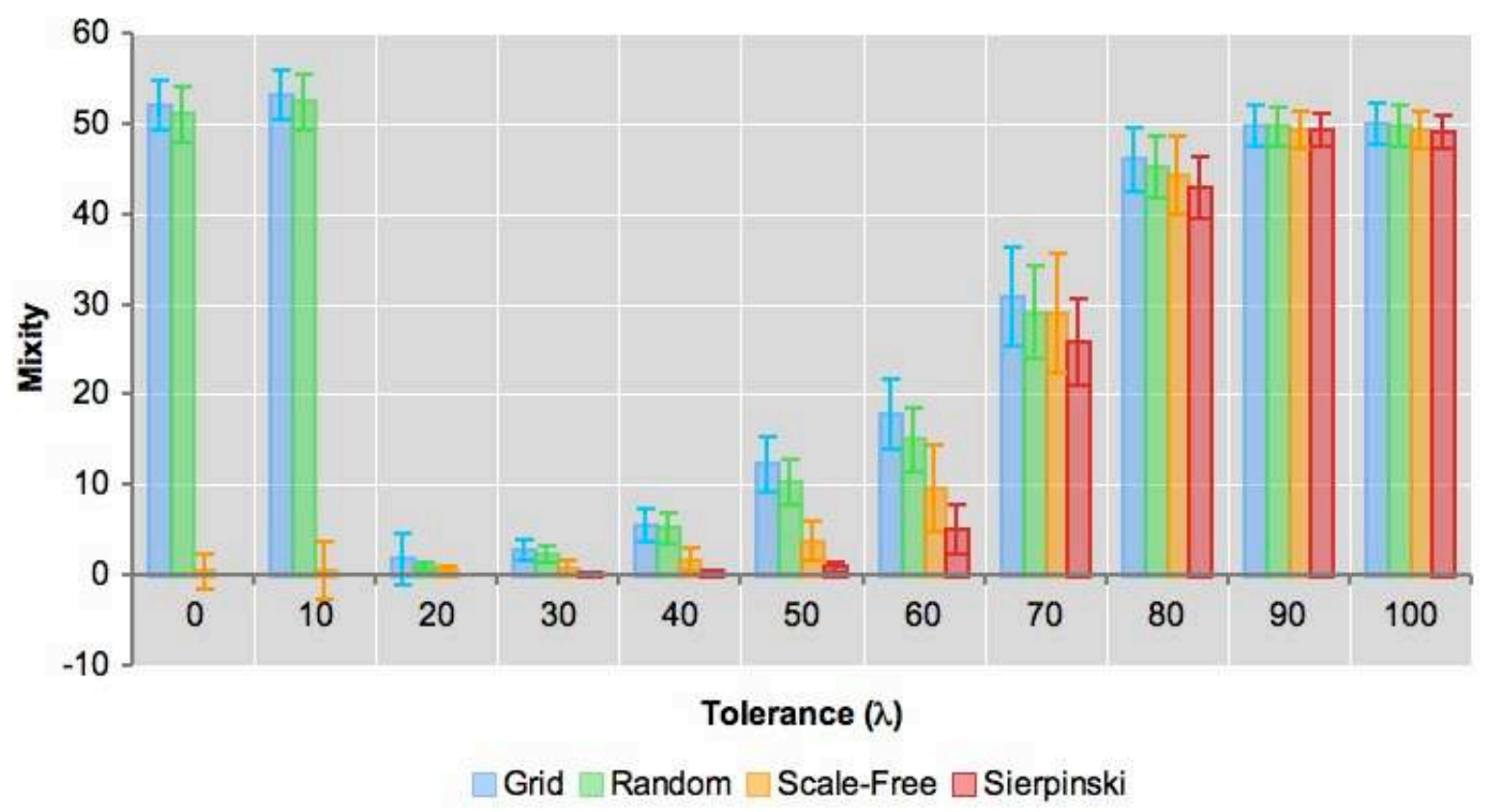

Figure 6. Average mixity index and its standard-deviation (1000 simulations) for each of the four networks, with noise in the system $(\mathrm{N}=0.1)$

One sees immediately two interesting phenomena. First, introducing a small amount of noise in the model improves its capacity to reach equilibrium for low tolerance values $(\lambda<40)$. For scale-free and Sierpinski networks, convergence is systematic even when tolerance is null $(\lambda=0)$. Therefore, adding a small amount of noise transforms Schelling's model in an optimization algorithm, able to find spatial patterns respecting heavy constraints on parameter $\lambda$. The underlying topology reinforces this capacity, especially when networks are highly interconnected (presence of cliques). Furthermore, this capacity is extraordinarily targeted: the course of the model doesn't seem to be affected by this small amount of noise, unless the second phase transition is reached $(\lambda=30)$. Figure 7 underlines clearly this property for the Sierpinski network: whatever the level of noise added, results are not significantly affected, until $\lambda>30$. Then, for $\lambda=30$ and $\lambda=20$, any amount of noise allows reaching equilibrium while the standard Schelling's model doesn't. As $\lambda$ decreases further on $(\lambda<20)$, a new state appears for $\mathrm{N}=1$ (i.e. $100 \%$ of unsatisfied agents move to any vacant node whatever their expected utility there), characterized by never ending movements of unsatisfied agents. It may be noticed that this chaotic state appears only when $\mathrm{N}=1$, i.e. when the system is populated with "knee-jerk" agents, moving to any vacant node as soon as they feel unsatisfied. Therefore, preserving an even small amount of "opportunist" Schelling like agents is sufficient to let the system converge towards equilibrium. 


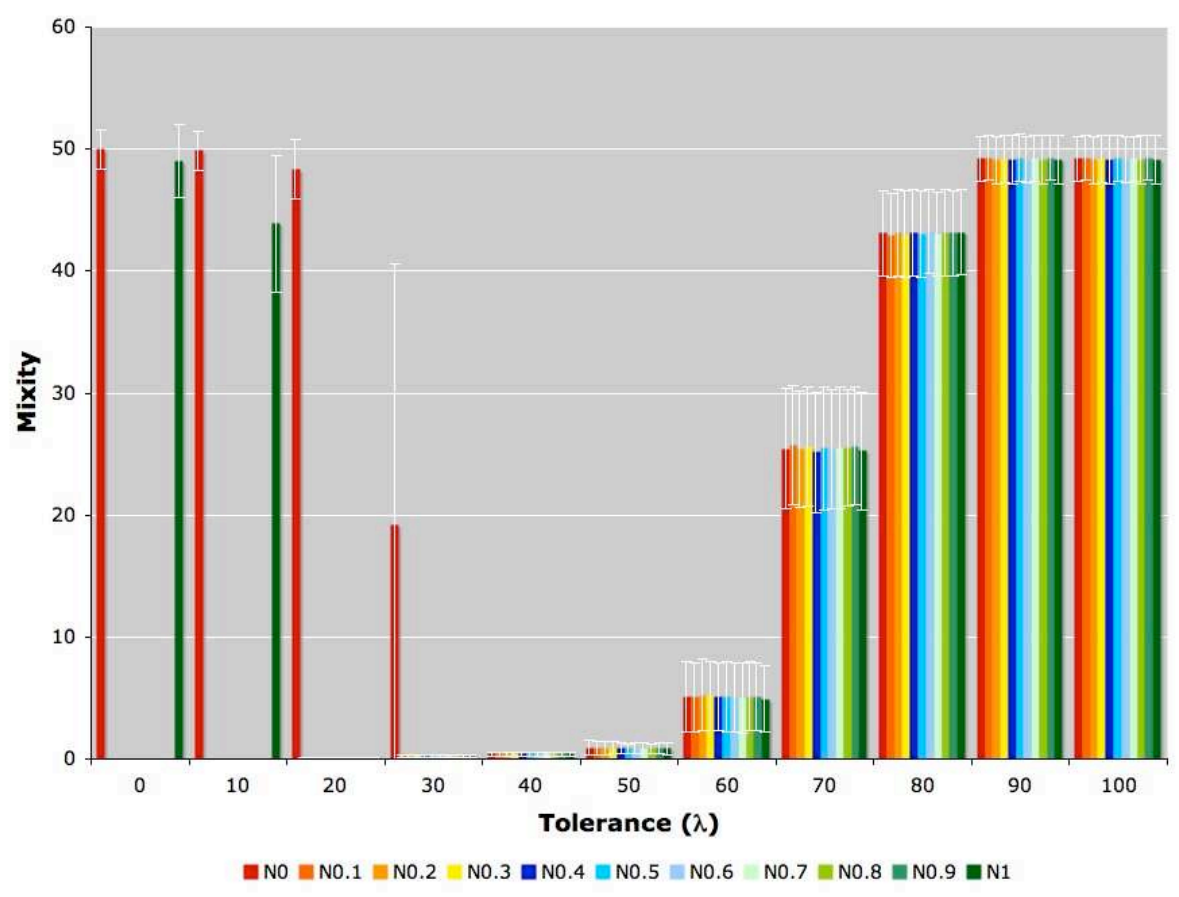

Figure 7. Mixity values reached for various noise levels, for the Sierpinski Network [density of occupied nodes $80 \%$ ]

\section{Sensitivity to initial conditions and network effects}

In this "tipping" model, segregation is then characterized as a stochastically stable state that tends to emerge and persist in the long run regardless of the initial state (Zhang, 2004). However, depending on the initial configuration of agents, their random selection and the succession of events (path dependency), very different equilibrium can be reached. In that sense, despite its global robustness, Schelling's model is also sensitive to initial conditions: different initial random patterns of agents may lead to very different final patterns. However, this sensitivity depends also on the value of the tolerance threshold used and its proximity to a stable or unstable zone of the parameters' space. Indeed, as illustrated by Figure 2, variability of final mixity index varies with tolerance value $\lambda$ and reaches the highest values for $\lambda=30$ and to a lesser extend for $\lambda=70$ (phase transitions). Between these two values, final configurations obtained differ essentially in their details, as global state - measured by mixity index - is quite stable. However, the situation is completely different when $\lambda=30$. Indeed, as figure 2 and table 2 suggest, this zone of the parameters' space is highly unstable and initial random distributions of agents can lead to very different configurations, depending on the capacity of the system to converge towards an equilibrium or to remain in a "frozen state". Figure 8 illustrates this phenomenon, which can be seen as a bifurcation: from given random initial distributions of agents and without added noise, system may converge towards a completely segregated configuration (mixity $<2 \%$ for both networks) or remain in a frozen state characterized by a high mixity index (mixity $>40 \%$ for both networks). 

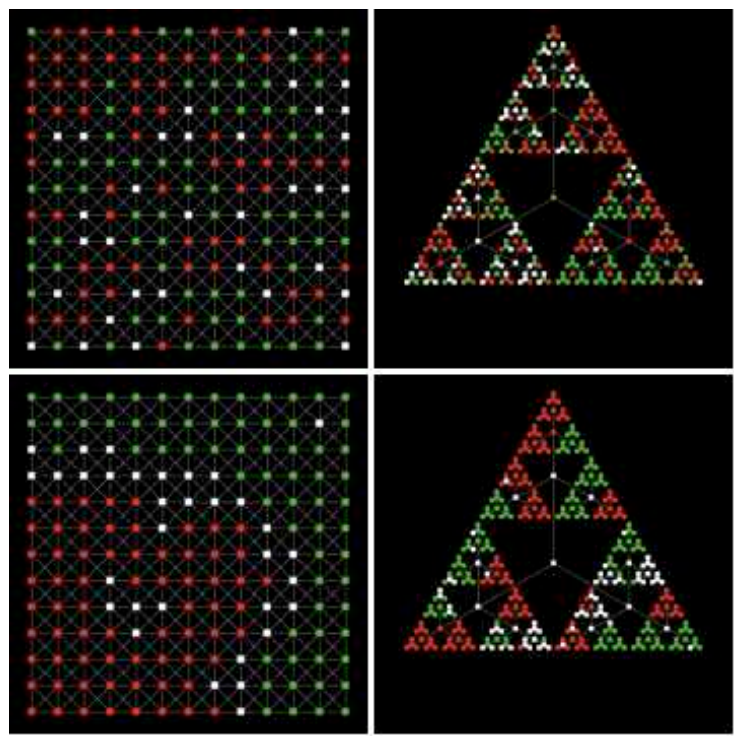

Figure 8. Example of bifurcation at work when $\lambda=30$ [Noise $=0$; Density $=80 \%$ ]

Therefore, when $\lambda=30$ Schelling's model is very sensitive to initial conditions and is consequently highly unstable, whatever the underlying network used. It may be noticed that adding a small amount of noise allows mastering this issue, as figure 5 and previous developments show. However, in addition to these global characteristics and adding just a limited amount of noise, one can also reveal a specific behavior of the model on Sierpinski network, characterized by a well-defined hierarchy of nodes. Indeed, as simulations point out (figure 9), the final state of major central nodes after convergence depends closely on the tolerance value.
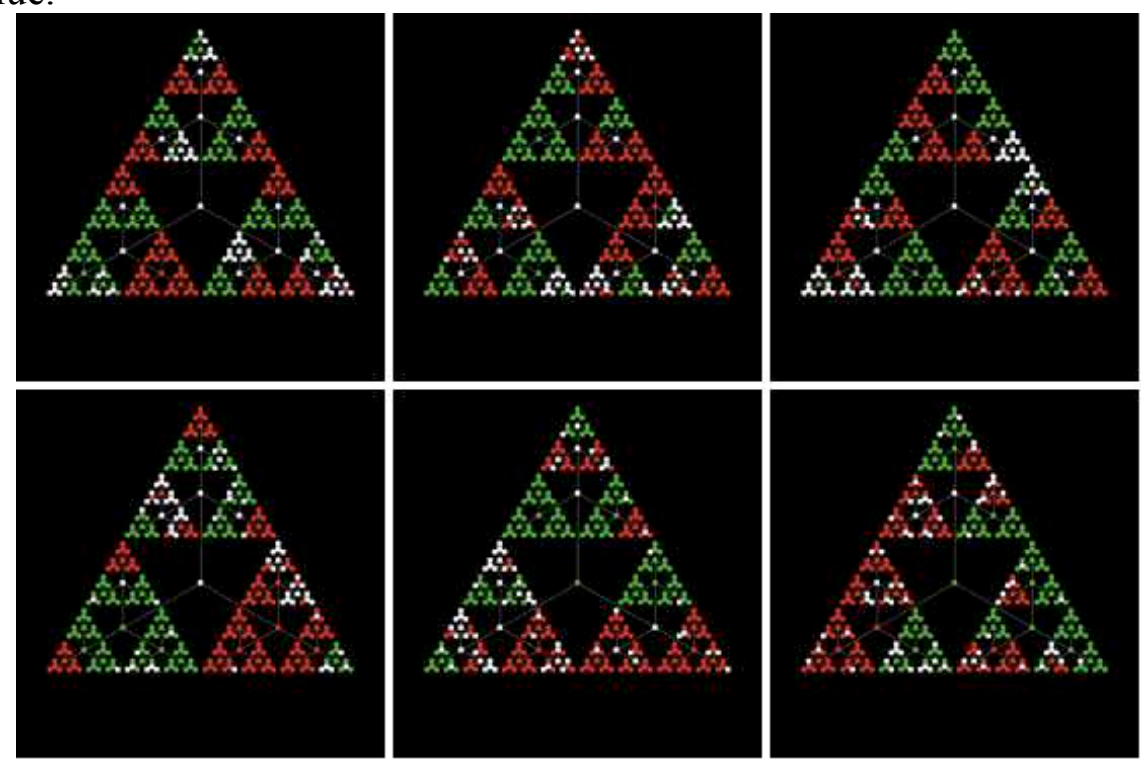

Figure 9. Influence of nodes' centrality on their final state $[$ Noise $=0.1$; Density $=80 \%$; $\lambda$ varies from 0 (upper left corner) to 50]

At any time during the simulation, nodes are characterized by one of the three possible states (vacant; occupied by an agent of color x; occupied by an agent of color y). On a completely homogeneous network, it is hardly possible to predict the final value of a given node. However, for Sierpinski network and for low tolerance values $(\lambda<30)$, this is not true anymore: whatever their initial state, central nodes almost systematically converge towards 
the same final state (vacant), separating cliques of homogeneously colored agents. Therefore, for highly hierarchised networks such as Sierpinski network, some nodes display very specific behavior depending on their topological situation. Space does make a difference, both at global and local levels.

\section{A global model}

As figure 6 suggests, mixity can be defined as a step function of tolerance $(\lambda)$, each underlying network having its own signature. Therefore, given the existence of two asymptotes (mixity $=0$ and mixity $=50$ ) and of an acceleration and deceleration phases between them, a three parameters logistic model may characterize this relation in a synthetic form:

$$
y= \begin{cases}50 & \text { if } \sum_{i=1}^{m} U_{i}<|A| \\ \frac{\gamma}{1+e^{-\alpha-\beta X}} & \text { if } \sum_{i=1}^{m} U_{i}=|A|\end{cases}
$$

with $y=$ Mixity and $x=$ Tolerance $(\lambda)$. Estimating parameters $\{\alpha, \beta, \gamma\}$ for each of the four networks leads to very tight fits $\left(\mathrm{R}^{2}>0.99\right)$. Figure 10 shows the various S-curves obtained.

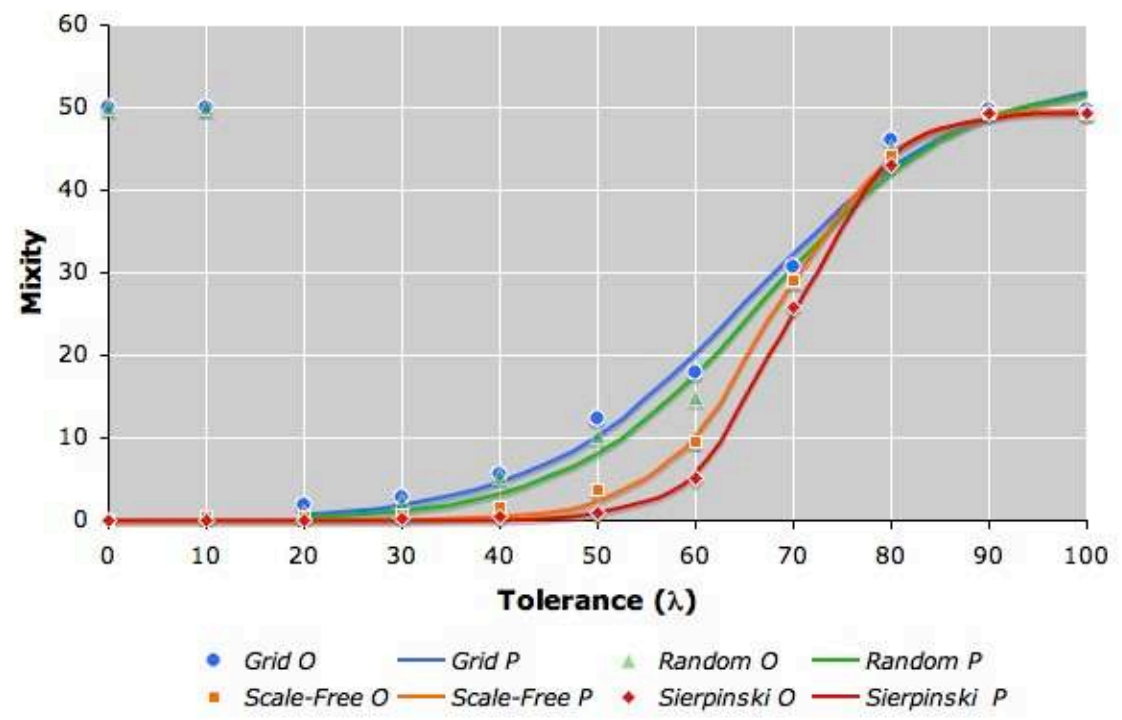

Figure 10. Logistic models fitted, for converging tolerance values $[$ Noise $=0.1$; in the legend " $O$ " is for observed (from simulation) and " $P$ " for predicted (model fitting)]

The parameters obtained (table 3) - and especially $\{\alpha, \beta\}$ as $\gamma$ is a scale parameter - allow comparing in a very synthetic manner Schelling's model behavior on various underlying structures. The surface between the two families of curves (grid and random versus scale-free and Sierpinski), coupled with the step-like structure of the logistic fits, give a precise idea of the capacity of the latter networks to accelerate and amplify Schelling like segregation. 


\begin{tabular}{|c|cccc|}
\hline Parameter & \multicolumn{4}{|c|}{ Grid Network Random Network Scale-Free Network Sierpinski Network } \\
\hline $\boldsymbol{\alpha}$ & -6.064 & -6.763 & -11.319 & -14.299 \\
$\boldsymbol{\beta}$ & 0.092 & 0.101 & 0.166 & 0.205 \\
$\boldsymbol{\gamma}$ & 54.019 & 53.632 & 50.076 & 49.406 \\
\hline \hline $\mathbf{R}^{\mathbf{2}}$ & 0.991 & 0.992 & 0.999 & 0.999 \\
\hline
\end{tabular}

Table 3. Parameters estimates and goodness-of-fit statistics $\left(R^{2}\right)$ for each network

These results suggest that for a given underlying topology (network and degree) and by introducing a small amount of noise (with its conservative properties suggested), we should be able to characterize and even anticipate Schelling's model behavior. On large networks, characterizing real cities, this could make a difference as the computation burden involved by simulation may exceed our capacities. Combined with further knowledge on local segregation dynamics related with cliques, these results might contribute assessing urban planning policies.

\section{Conclusion}

So far, several conclusions can be drawn from our simulations. Firstly, and confirming previous works, Schelling's segregation process is robust and happens on very different underlying structures, even far away from the homogeneous grid initially used by Thomas Schelling. Secondly, this model is also very robust to random fluctuations. Indeed, introducing a small amount of noise in the model dynamic under the form of a small proportion of "knee-jerk" agents, moving to any vacant node as soon as they feel unsatisfied whatever their expected utility, has very limited impact on its global behavior. However, for specific tolerance values, such add-on allows reaching equilibrium solutions that were not attainable with the standard version. Under this slight modification, we show that the global behavior of Schelling's model can be formalized in a simple thus precise way, with a three parameters logistic model.

These fundamental properties of Schelling's model then allowed us showing by simulation that, contrarily to previous conclusions (Fagiolo et al, 2007; Fossett and Dietrich, 2009), space does matter in this model. More precisely, network topology interferes with its dynamics, by accelerating and reinforcing its effects. Segregation occurs more rapidly on hierarchised networks characterized by cliques than on random and regular ones. Furthermore, such interconnected structures also authorize intolerant behaviors: equilibrium can indeed be reached with highly segregationists agents (tolerance weak or null), as soon as a small proportion of non opportunist agents is introduced in the system. Preliminary investigations led on "real" cities confirm these theoretical results. In (Moreno et al, 2009), the Schelling model was applied to a large network (120 000 nodes), each building being embedded in an anisotropic neighborhood, defined using accessibility and graph-based algorithms, in the same spirit we did in the present work. Clique effects were also identified, though in a less systematic way due to the computation burden involved by the network' size. These results may have important public policy and urban planning implications as they suggest that actual and planned geometries of cities may not only accelerate and reinforce Schelling' segregation processes but may also contribute favoring segregationist behaviors. Therefore, fatalism should be banned and laissez-faire should be fought: public policy and urban planning have their role to play. Limiting undesirable self-reinforcement processes may be possible and should be part of any ambitious urban politics.

Moreover, this work highlights the need for more integrated approaches of urban systems. Indeed, as (Frankhauser, 2008) shows, hierarchical and interconnected urban architectures prove useful on some aspects. Especially fractal geometries, which may benefit to cities and 
citizens, as they allow maintaining "a social mix by means of a higher local variability of densely and less densely populated zones and, on the other hand, to preserve huge empty zones in the neighborhood of urbanized areas, which may be imagined as natural reserves, agricultural zones or simply leisure areas offering rural amenities" [pp. 239]. Our simulations show evidence that such gains may be compensated by losses in terms of social mixity and integration. Based on these results, it seems obvious that more integrated approaches of urban systems should be encouraged and privileged in our more than ever urgent quest for sustainable cities. Among the challenges put at the forefront by this classical model, one finds news echoes here: can we imagine cities' shapes and forms able to limit Schelling's segregation process? Obviously, one cannot focus only on individual preferences, as suggested by (Clark and Fossett, 2008). Economic constraints, socio-economic context and precise survey-based knowledge of the precise mechanisms at work should be incorporated incrementally in the model, in order to gain new insights on its behavior in more complex situations, not limited to positive feedbacks and self-reinforcement processes. Carefully introducing heterogeneity in agent-based models, both at the level of agents' attributes and behaviors but also in the way we define agents' environment is therefore a key issue the community should handle more vigorously, in order to improve our understanding of cities dynamics.

\section{References}

Barabási, A, Albert R 1999, "Emergence of scaling in random networks", Science 286509 512

Batty M 2008, "The size, scale, and shape of cities", Science $319769-771$

Clark A, Fossett M 2008 "Understanding the social context of the Schelling segregation model”, PNAS 105-11 4109 - 4114

Dall'Asta L, Castellano C, Marsili M 2008, "Statistical physics of the Schelling model of segregation" Journal of Statistical Mechanics L07002 http://www.iop.org/EJ/article/17425468/2008/07/L07002/jstat8_07_107002.html

Fagiolo G, Valente M, Vriend NJ 2007, "Segregation in Networks", Journal of Economic Behavior and Organization 64316 - 336

Floyd RW 1962, "Algorithm 97: Shortest Path" Communications of the ACM 56345

Fossett M, Dietrich DR 2009, "Effects of city size, shape, and form, and neighborhood size and shape in agent-based models of residential segregation: are Schelling-style preference effects robust?" Environment and Planning B: Planning and Design 36(1) 149 - 169

Frankhauser P 2008, "Fractal geometry for measuring and modeling urban patterns", in The Dynamics of Complex Urban Systems, Eds S Albeverio, D Andrey, P Giordano, A Vancheri, (Physica-Verlag, Heidelberg) pp $213-243$

Gauvin L, Vannimenus J, Nadal JP 2009, "Phase diagram of a Schelling segregation model", The European Physical Journal B, 70293 - 304

Jiang B 2007, “A topological pattern of urban street networks: Universality and peculiarity", Physica A, 384647 - 655

Keeling MJ 1999, "The effects of local spatial structure on epidemiological invasions", Proceedings of the Royal Society London B, 266859 - 867

Laurie AJ, Jaggi NK 2003, “The role of 'vision' in neighbourhood racial segregation: a variant of the Schelling segregation model”, Urban Studies 402687 - 2704

Moreno D, Badariotti D, Banos A 2009, Integrating morphology in urban simulation through reticular automata, in European Handbook of Theoretical and Quantitative Geography, Eds F Bavaud, C Mager (FGSE, Lausanne) pp 261 - 309 
Newman MEJ, Strogatz SH, Watts DJ 2001, "Random graphs with arbitrary degree distributions and their applications", Physical Review E 64026118

Pancs R, Vriend NJ 2007 "Schelling's spatial proximity model of segregation revisited", Journal of Public Economics 911 - 24

Pollicot M, Weiss H 2001, The dynamics of schelling-type segregation models and a nonlinear graph Laplacian variational problem, Advances in Applied Mathematics 27(1) 17 40

Schelling, T 1969, "Models of segregation", American Economic Review, Papers and Proceedings, 59488 - 493

Schelling, T 1978, Micromotives and Macrobehavior (Norton, New York)

Song C, Havlin S, Makse H 2006 "Origins of fractality in the growth of complex networks", Nature Physics 2275 - 281

Stauffer D, Solomon S 2007, "Ising, Schelling and Self-Organising Segregation", European Physical Journal B 57(4) 473 - 479

Vinkovic D, Kirman A 2006 "A physical analogue of the Schelling model", PNAS 103(51) $19261-19265$

Watts D, Strogatz S 1998, "Collective dynamics of small-world networks", Nature 393(4) $440-442$

Zhang J 2004, "A dynamical model of residential segregation", Journal of Mathematical Sociology $28147-170$ 